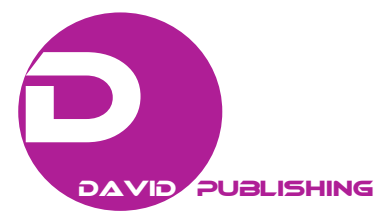

\title{
Small Bowel Intussusception in a Patient with Von Recklinghausen's Neurofibromatosis
}

\author{
Hamri Asma, Narjis Youssef and Benelkhaiat Ridouan \\ Department of Surgery, Ibn Tofail Hospital, MohamedVI University Hospital, University of Medicine and Pharmacy, Marrakech
}

40000, Morocco

\begin{abstract}
Neurofibromatosis type 1 (NF1) is one of the most common genetic disorders. Mutations of the NF1 gene lead to abnormal tumor suppression. Consequently, patients with NF1 have an increased prevalence of benign and malignant neoplasms throughout the body. Neuroendocrine tumor is one of many forms of neoplasm with neurofibromatosis-1 (NF-1). The gut isaffected in $30 \%$ of cases. Case report: A 43-year-old woman with NF-1 had spontaneously resolutive subocclusion, slimming and right iliac fossa mass. She was admitted to our hospital for further examination. An abdominal contrast-enhanced computed tomography scan demonstrated an intestinal invagination and mild intestinal distension. A bowel resection, carrying the affected bowel and the cecum, was performed. The anatomo-pathology study was Carcinoma not differentiated ulcerated on the surface (the muscular, the sub-serous) with vascular emboli and perinervous catheterization. Of healthy excision with ganglion metastases $(5+/-15)$. The appearance is in favor of a high-grade neuroendocrine tumor. The tumor was diagnosed as NET G3. The postoperative sequences are simple. The patient had no further symptoms in the 12 months after the chemotherapy
\end{abstract}

Key words: Neuroendocrine tumor, von Recklinghausen's disease.

\section{Background}

Neurofibromatosis type 1 (NF1) is one of themost fascinating and common human mendelian disorders, affecting approximately one in 3000 persons [1]. Benign and malignant neoplasms may arise in the abdomen in both pediatric and adult patients with NF1. The abdominal neoplasms in NF1 can be divided into five basic categories: neurogenic tumors, neuroendocrine tumors, nonneurogenic gastrointestinal mesenchymal tumors, embryonal tumors, and miscellaneous tumors [2].

\section{Case Report}

A 43-year-old woman with NF-1 (Fig. 1) had spontaneously resolutive subocclusion, slimming and right iliac fossa mass. She was admitted to our hospital for further examination. An abdominal contrast-enhanced computed tomography scan

Corresponding author: Benelkhaiat Ridouan, Ph.D., research field: surgery. demonstrated an intestinal invagination and mild intestinal distension. Surgical exploration: the small intestine Intussusception (entero-enteric) with tumor (Fig. 2). A bowel resection, carrying the affected bowel and the cecum, was performed. The anatomo-pathology study was Carcinoma not differentiated ulcerated on the surface (the muscular, the sub-serous) with vascular emboli and perinervous catheterization. Of healthy excision with ganglion metastases $(5+/-15)$. The appearance is in favor of a high-grade neuroendocrine tumor. The tumor was diagnosed as NET G3. The postoperative sequences are simple. The patient had no further symptoms in the 12 months after the chemotherapy.

\section{Discussion}

NF1 belongs to a group of disorders referred to as phakomatoses. These disorders (NF1, neurofibromatosis type 2, tuberous sclerosis, Sturge-Weber syndrome, and neurocutaneous melanosis) have selective involvement of tissues of 


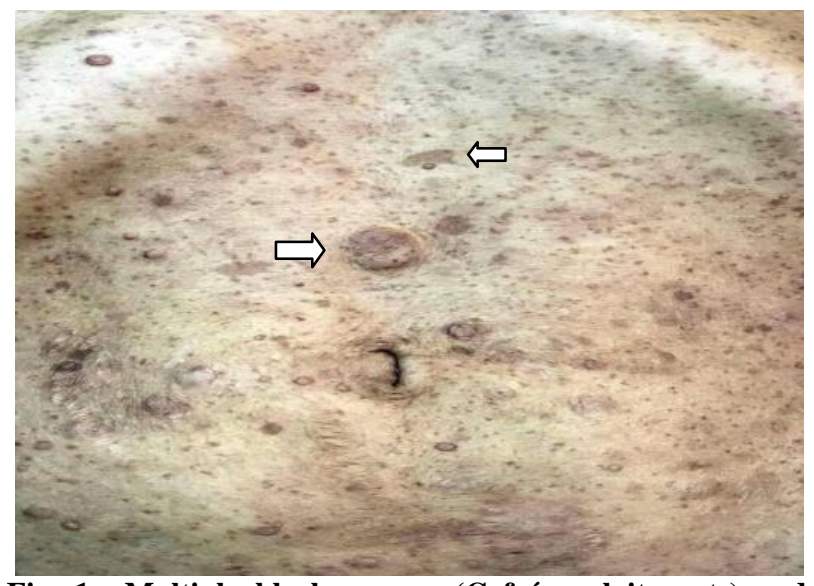

Fig. 1 Multiple black arrows (Cafe' au lait spots) and sessile cutaneous neurofibromas on the anterior abdomen.

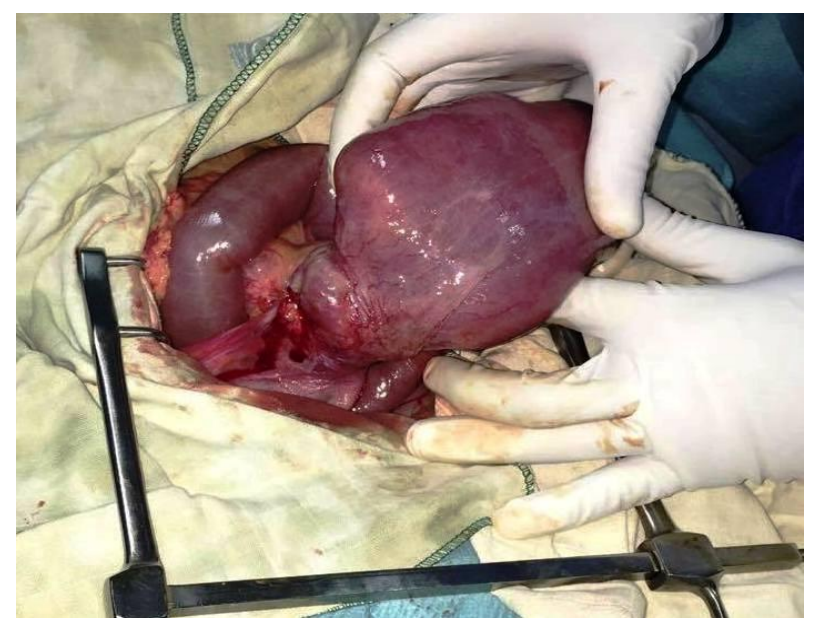

Fig. 2 The small intestine Intussusception (entero-enteric).

ectodermal origin (central nervous system, eye, and skin). NF1 is a complex disease resulting from a spectrum of mutations that may occur at many locations along the large, complex NF1 gene, which is located on chromosome 17. All of these disorders, with the exception of Sturge-Weber syndrome, have an autosomal dominant inheritance pattern. NF1 affects all races and both sexes equally, occurring in the population with a prevalence of approximately one in
3000 persons [3]. Only $50 \%$ of patients with NF1 have a positive family history. In the remaining $50 \%$ of patients, the disorder represents a sporadic new mutation, a reflection of the high mutation rate of the NF1 gene. Although NF1 has high penetrance, expression is quite variable. Many patients with NF1 are only mildly affected. Gastrointestinal carcinoids are endocrine neoplasms that originate from mucosal or submucosal endocrine cells. Carcinoids are more common in patients with NF1 than in the general population. Carcinoids in patients with NF1 show the same range of malignancy as those found in the general population [4].

\section{Conclusion}

Any abdominal pain in a patient with neurofibromatosis, tumoral process must be seek .

\section{References}

[1] Huson, S. M., Compston, D. A., Clark, P., and Harper, P. S. 1989. "A Genetic Study of von Recklinghausen Neurofibromatosis in South East Wales. I. Prevalence, Fitness, Mutation Rate, and Effect of Parental Transmission on Severity." J. Med. Genet. 26 (11): 704-11.

[2] Angela, D. L., Nandini, P., Nancy, D., Robert, M. A., Markku, M., and Leslie, H. S. 2005. "Abdominal Neoplasms in Patients with Neurofibromatosis Type 1: Radiologic-Pathologic Correlation.” Radiographics 25 (2): 455-80.

[3] Von Recklinghausen, F. D. 1882. On Multiple Fibromas in the Skin and their Relationship to Multiple Neuromas. Berlin, Germany: Hirschwald. (in German)

[4] Xu, G. F., O'Connell, P., Viskochil, D., Cawthon, R., Robertson, M., Culver, M., Dunn, D., Stevens, J., Gesteland, R., and White, R. 1990. "The Neurofibromatosis Type 1 Gene Encodes a Protein Related to GAP.” Cell (62): 599-608. 